\title{
Magnetization Studies of Antiferromagnetic Interlayer Coupling in EuS-SrS Semiconductor Multilayers
}

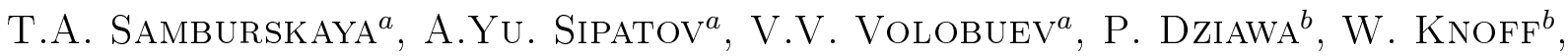 \\ L. KOWALCZYK ${ }^{b}$, M. SzOT ${ }^{b}$ AND T. STORY ${ }^{b}$ \\ ${ }^{a}$ National Technical University "Kharkov Polytechnic Institute", 21 Frunze Str., 61002 Kharkov, Ukraine \\ ${ }^{b}$ Institute of Physics, Polish Academy of Sciences, al. Lotników 32/46, 02-668 Warsaw, Poland
}

(Received March 27, 2013; in final form April 12, 2013)

\begin{abstract}
Temperature and magnetic field dependence of magnetization of EuS-SrS multilayers grown epitaxially on $\mathrm{KCl}$ (001) substrate is experimentally studied by superconducting magnetometry technique. In these lattice-matched semiconductor heterostructures EuS layers are ferromagnetic quantum wells whereas SrS layers are nonmagnetic spacer barriers. The multilayers composed of EuS layers with thickness $3.5-5 \mathrm{~nm}$ and SrS layers (thickness $0.5^{-}$ $10 \mathrm{~nm}$ ) exhibit ferromagnetic transition at $17 \mathrm{~K}$. In the multilayers with ultrathin $\operatorname{SrS}$ spacers $(0.5-1 \mathrm{~nm})$ a nonmonotonic temperature dependence of magnetization as well as a characteristic switching in magnetic hysteresis loops is observed. These experimental findings are explained considering antiferromagnetic interlayer coupling between ferromagnetic EuS layers via nonmagnetic SrS spacers. The strength of this coupling is determined based on model magnetization calculations.
\end{abstract}

DOI: $10.12693 /$ APhysPolA.124.133

PACS: 75.20.Ck, 75.30.Et

\section{Introduction}

Europium chalcogenides (EuO, EuS, EuSe, and EuTe) constitute a family of model magnetic semiconductors with nearest-neighbor and next-nearest-neighbor Heisenberg exchange interactions of different sign resulting in either ferromagnetic (in EuO and EuS) or antiferromagnetic (in EuTe) order. EuSe is known to possess a more complex magnetic phase diagram. EuS crystallizes in the rock-salt structure with the lattice parameter $a_{0}=0.5967 \mathrm{~nm}$. It is an insulating material with the energy gap of $1.65 \mathrm{eV}$. Ferromagnetic transition in EuS bulk crystals takes place at the Curie temperature $T_{\mathrm{C}}=16.5 \mathrm{~K}[1]$.

Several semiconductor materials (e.g., $\mathrm{PbS}, \mathrm{SrS}$, and YbSe) provide excellent lattice matching to ferromagnetic EuS and permit the growth of high crystal quality, epitaxial ferromagnetic-nonmagnetic multilayers composed of semiconductor materials only [2]. In the best studied EuS-PbS heterostructures the nonmagnetic PbS layer also crystallizes in the rock-salt structure with the lattice parameter $a_{0}=0.5936 \mathrm{~nm}[3,4] . \mathrm{PbS}$ is a narrow gap $(0.3 \mathrm{eV})$ semiconductor that in EuS-PbS multilayers serves as a nonmagnetic quantum well with ferromagnetic EuS barriers [4]. For EuS layers thinner than about $3 \mathrm{~nm}$ the Curie temperature is reduced as compared to the reference value in bulk crystals due to the size-effect. It can be explained in a simple mean field model by accounting for the reduced number of magnetic neighbors of Eu ions [3]. These multilayers are thermally strained due to the difference between the thermal expansion coefficients of the layers and the $\mathrm{KCl}$ or $\mathrm{BaF}_{2}$ substrates. The strain effect is particularly strong for EuS layers deposited on $\mathrm{KCl}$ substrate. It leads to an in-plane biaxial compression of the EuS lattice and results in an experimentally observed increase of the Curie temperature $T_{\mathrm{C}}$ by about $2 \mathrm{~K}[3,4]$.

In contrast to EuS-PbS heterostructures, in EuS-SrS multilayers, the ferromagnetic layers of EuS form quantum wells separated by wide-band-gap (about $7 \mathrm{eV}$ ) SrS nonmagnetic barriers. SrS also crystallizes in the rock-salt structure with the lattice parameter $a_{0}=0.602 \mathrm{~nm}$ matching very well to EuS $[2,5]$. In previous magnetization studies of EuS-SrS multilayers grown on $\mathrm{KCl}$ we have determined the ferromagnetic transition temperature $T_{\mathrm{C}}$, magnetic anisotropy (easy magnetization axis located in-plane along [110] direction) and other magnetic characteristics such as coercive field and saturation magnetization [5]. We found no evidence for interlayer exchange coupling (IEC) via SrS spacers of thickness down to $1 \mathrm{~nm}$. However, a strong evidence for antiferromagnetic IEC was found in recent neutron reflectivity experiments carried out on EuS-SrS multilayers grown by the same technological method [2].

The effect of antiferromagnetic IEC provides one of the main building blocks in designing new spintronic devices. The antiparallel orientation of magnetization vectors of neighboring magnetic layers (with zero total magnetic moment) can be switched by relatively weak external magnetic field to the parallel orientation with full ferromagnetic magnetization of both layers. This switching of magnetization vectors is sensed by either magnetoresistive (GMR) or tunelling (TMR) magnetoelectronic structures. The phenomenon of IEC is well established in metallic spintronic systems in which the RudermanKittel-Kasuya-Yosida (RKKY)-type mechanism is usually effective. It exploits spin polarization of free charge carriers in the spacer layer. In all-semiconductor ferromagnetic heterostructures with negligible carrier concen- 
tration the IEC effects were observed e.g. in EuS-PbS [2, 6-9] and (Ga,Mn)As-GaAs [10-12] multilayers by both neutron reflectivity and magnetization measurements. Apart from the modification of the RKKY mechanisms for two-dimensional semiconductor heterostructures with low carrier concentration [13, 14], theoretical proposals specific for nonmetallic systems were also put forward. These new mechanisms rely on spin polarization of valence band electrons and interband electronic transition [15] or involve magnetization orientation sensitive contributions to electronic band structure in the entire first Brillouin zone [16].

In this work we apply superconducting magnetometry method to experimentally study the antiferromagnetic IEC in EuS-SrS-EuS trilayers with ultrathin SrS spacers of thickness down to only two monolayers. By quantitative modeling of the magnetic field dependence of magnetization we also determine the relevant interlayer exchange integral. We apply the experimental procedure and model calculations successfully used by us previously in the studies of antiferromagnetic IEC in closely related EuS-PbS multilayers [7].

\section{Technology and experiment}

EuS-SrS multilayers were grown by high vacuum $\left(10^{-7}\right.$ Torr $)$ deposition of EuS and SrS molecules on freshly cleaved (001) surface of monocrystalline $\mathrm{KCl}$ substrate. Prior to deposition of the multilayer about 20$40 \mathrm{~nm}$ thick $\mathrm{PbS}$ buffer layer was grown. Electron guns were used to evaporate EuS and SrS from solid state compound sources whereas for $\mathrm{PbS}$ a resistively heated tungsten boat was used. The substrate temperature during growth was $250-300^{\circ} \mathrm{C}$. The thickness of the layers was monitored in situ with a calibrated quartz resonator. After the growth it was also independently checked in the $\mathrm{X}$-ray diffraction (XRD) analysis of periodic multilayers (superlattices). The crystal structure investigations by the transmission electron microscopy (TEM) and the XRD techniques showed that EuS and SrS are monocrystalline (001) crystallographically oriented layers with the $\mathrm{X}$-ray rocking curve full width at half maximum of about $0.02^{\circ}-0.04^{\circ}$. For EuS-SrS superlattices the XRD spectra show clear satellite peaks even up to the fourth order (Fig. 1), indicating the well-defined planar structures of ultrathin magnetic and nonmagnetic layers with sharp interfaces. Due to very good lattice match between EuS and $\mathrm{SrS}$ these semiconductor materials can be grown as pseudomorphically strained structures up to the total thickness of about $200 \mathrm{~nm}$ which exceeds the total thickness of any multilayer studied in this work. Previous studies showed that the interdiffusion at the EuS/ $\mathrm{SrS}$ interface is low and corresponds to an intermixing of only about one monolayer [2]. In EuS-SrS multilayers and trilayers studied in this work the layer thickness covered the range 0.5-10 nm for nonmagnetic SrS spacer and 3.5-5 $\mathrm{nm}$ for ferromagnetic EuS layers.

Magnetic properties of EuS-SrS multilayers were examined by superconducting (SQUID) magnetometry

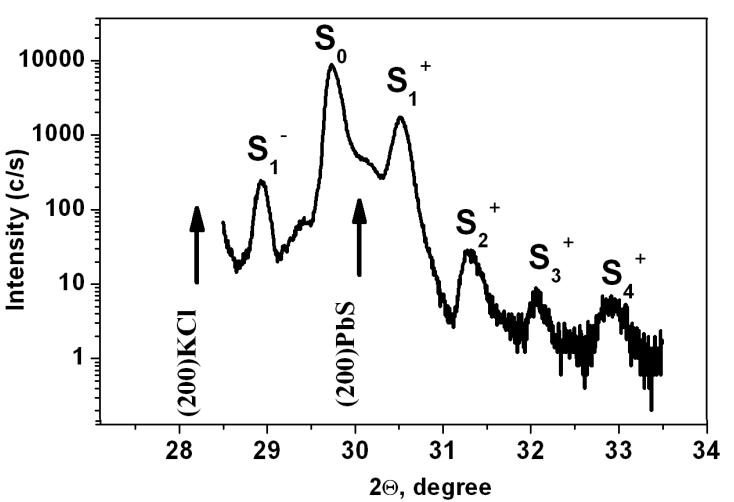

Fig. 1. X-ray diffraction spectrum $(\theta-2 \theta$ scan $)$ of the superlattice $10 \times \quad[\operatorname{EuS}(3.5 \quad \mathrm{~nm})-\mathrm{SrS}(8 \mathrm{~nm})] /$ $\mathrm{PbS}(20 \mathrm{~nm}) / \mathrm{KCl}(001)$ near $(200)$ Bragg reflection. $S_{n}$ indicate the superlattice satellite reflections.

method in the temperature range $T=4-35 \mathrm{~K}$ covering both ferromagnetic and paramagnetic temperature regions. The weak external magnetic field $H \leq 300$ Oe was applied in the (001) plane of the multilayer along [110] crystal direction being an easy magnetization axis. In external magnetic field of 300 Oe applied in the plane of the layer the ferromagnetic layers of EuS are expected to reach magnetic saturation state $[2,3,5]$. In relatively weak magnetic fields applied in our studies the diamagnetic contribution of the $\mathrm{KCl}$ substrate is below $5 \%$ of the total magnetic moment of the multilayer whereas the diamagnetic contribution of $\mathrm{PbS}$ buffer layer is negligible.

\section{Discussion and conclusions}

In the studied here EuS-SrS heterostructures the temperature dependence of magnetization reveals a sharp transition to ferromagnetic state at the Curie temperature of $T_{\mathrm{C}}=17 \mathrm{~K}$. The strong magnetic shape anisotropy contribution (anisotropy field $H_{\mathrm{A}} \approx 15 \mathrm{kOe}$ ) results in the in-plane location of the magnetization vector. In the (001) layer plane we observe only weak magneto-crystalline anisotropy contribution $\left(H_{\mathrm{A}} \approx 0.1 \mathrm{kOe}\right)$ with four equivalent $\{110\}$ easy magnetization directions. The magnetization saturation values found experimentally in EuS layers at $T=5 \mathrm{~K}$ are in the range 700 $1150 \mathrm{emu} / \mathrm{cm}^{3}$. It is close to the theoretical value of $1180 \mathrm{emu} / \mathrm{cm}^{3}$ expected for the ideal EuS layer in which all the europium ions are in $\mathrm{Eu}^{2+}$ state and possess magnetic moment of 7 Bohr magnetons. The saturation magnetization of the order of $(70-90) \%$ of theoretical limit is frequently observed in ultrathin layers of $\mathrm{EuS}[3-5]$. It is mainly related to surface and interface morphology effects changing magnetic properties at the interface. The experimental error in determination of the saturation magnetization of a trilayer is rather large (15-20\%) due to limited precision of determination of thickness and planar dimensions of ultrathin layers.

In Fig. 2 the magnetization hysteresis loops for two EuS-SrS-EuS trilayers with different spacer thicknesses 


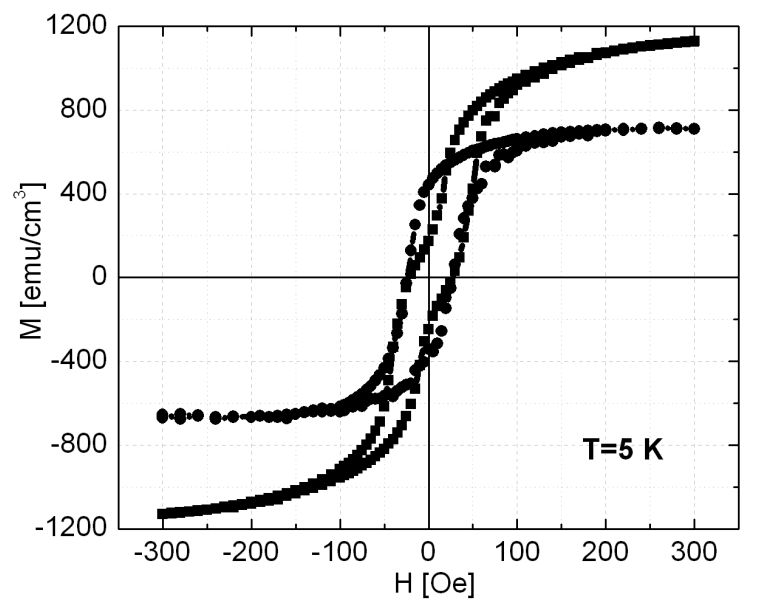

Fig. 2. Magnetization loops of $\operatorname{EuS}(3.5 \mathrm{~nm})-$ $\operatorname{SrS}(5.5 \mathrm{~nm})-\operatorname{EuS}(3.5 \mathrm{~nm})(\operatorname{dots})$ and $\operatorname{EuS}(3.5 \mathrm{~nm})-$ $\mathrm{SrS}(0.5 \mathrm{~nm})-\mathrm{EuS}(3.5 \mathrm{~nm})$ (squares) trilayers grown on $\mathrm{KCl}(001)$ substrate.

$d_{\mathrm{SrS}}=0.5 \mathrm{~nm}$ and $d_{\mathrm{SrS}}=5.5 \mathrm{~nm}$ are presented. The EuS layers are $3.5 \mathrm{~nm}$ thick in both cases. In contrast to a regular magnetization loop observed for the trilayer with the thick SrS spacer the trilayer with the thin SrS spacer exhibits a characteristic switching at the applied magnetic field of 25 Oe (Fig. 2) and 50 Oe (Fig. 3) with a strongly reduced remanent magnetization. These effects were observed by us in several trilayers but only for $\mathrm{SrS}$ spacers with the thickness $d_{\mathrm{SrS}}=0.5-1 \mathrm{~nm}$. Such step-like behavior can be attributed to the antiferromagnetic interlayer coupling of neighboring ferromagnetic EuS layers via nonmagnetic SrS spacer layer. The step-like behavior is a manifestation of the abrupt transition of magnetization vectors of EuS layers from one stable magnetic arrangement (antiparallel) to another (parallel).

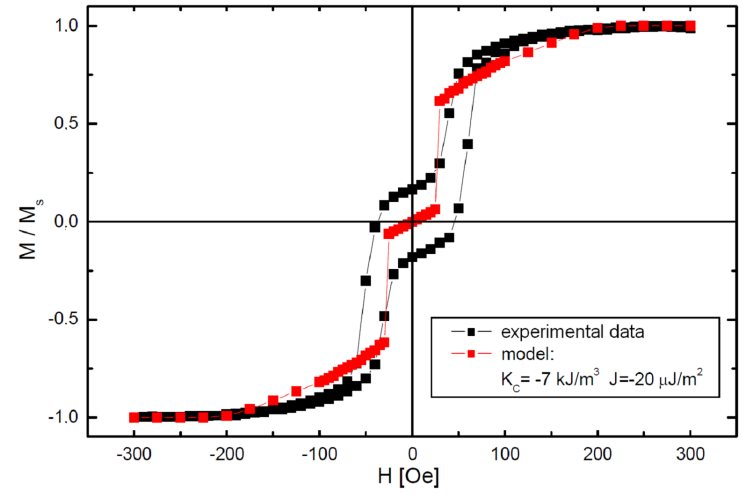

Fig. 3. Magnetization hysteresis loop for $\operatorname{EuS}(3.5 \mathrm{~nm})-\operatorname{SrS}(0.7 \quad \mathrm{~nm})-\operatorname{EuS}(3.5 \mathrm{~nm}) \quad$ trilayer and the result of model calculations taking into account Zeeman energy term, antiferromagnetic interlayer exchange coupling $(J)$, and cubic in-plane magneto-crystalline anisotropy $\left(K_{\mathrm{C}}\right)$. Hysteresis effects are not included in the model calculations.
To determine the strength of the IEC in EuS-SrS-EuS trilayers quantitatively we carried out model calculations of the total magnetization of a system of two ferromagnetic layers coupled antiferromagnetically. The interlayer exchange energy (per unit area) is given by the phenomenological expression: $E_{\mathrm{ex}}=-J M_{1} \boldsymbol{M}_{2}$. Magnetic energy density of each individual layer consist additionally of the Zeeman magnetostatic term $E_{\mathrm{H}}=$ $-\boldsymbol{H} \boldsymbol{M}$ and magnetocrystalline anisotropy term $E_{\mathrm{A}}=$ $K_{\mathrm{C}} \sin ^{2}(\varphi) \cos ^{2}(\varphi)$. Here $\varphi$ is the angle between the magnetization vector $\boldsymbol{M}$ and the easy axis of magnetization set as [110] crystal direction. Finding for each magnetic field $H \|[110]$ and temperature $T$ the global minimum of the total magnetic energy $E_{\mathrm{M}}(H, T)=$ $E_{\mathrm{H} 1}+E_{\mathrm{H} 2}+E_{\mathrm{A} 1}+E_{\mathrm{A} 2}+E_{\mathrm{ex}}$ we determined the magnetization vectors directions and their components on the external magnetic field $\left(M_{\mathrm{H}}\right)$, i.e. the physical quantity measured by the SQUID magnetometer. In Fig. 3 we present the example of such magnetization modeling for EuS-SrS-EuS system with the antiferromagnetic IEC integral $J$ and anisotropy constant $K_{\mathrm{C}}$ parameters indicated in the figure. The basic experimental features of the $M_{\mathrm{H}}(H)$ dependence are well reproduced in the calculations permitting the determination of the exchange integral $J$. This parameter directly influences the switching field and the saturation field in magnetization loops. It is known that such global minimum calculations of magnetization do not yield magnetic hysteresis effects. More detailed description and analysis of this model is given in Ref. [7].

Our model calculations yield the following results for the antiferromagnetic IEC in EuS-SrS-EuS trilayers with varying spacer thickness: $J=-15 \mu \mathrm{J} / \mathrm{m}^{2}$ for $d_{\mathrm{SrS}}=$ $0.7 \mathrm{~nm}, J=-20 \mu \mathrm{J} / \mathrm{m}^{2}$ for $d_{\mathrm{SrS}}=0.6 \mathrm{~nm}$, and $J=-11 \mu \mathrm{J} / \mathrm{m}^{2}$ for $d_{\mathrm{SrS}}=0.5 \mathrm{~nm}$. In agreement with our previous magnetometric studies of EuS-SrS multilayers [5], we found no evidence for the IEC in trilayers with SrS spacers thicker than $1 \mathrm{~nm}$. It is, most likely, related to theoretically predicted very rapid exponential decay of the strength of the IEC with the increasing spacer thickness [16]. Comparing our results to the reference EuS-PbS materials system one finds in EuS-SrS multilayers the antiferromagnetic IEC of comparable strength that corresponds to the external switching fields in the range 10-100 Oe. The precise quantitative analysis of the IEC integral in the case of ultrathin spacers is limited by various ferromagnetic bridges (pinholes) inevitably present in macroscopically large $\left(3 \times 3 \mathrm{~mm}^{2}\right)$ samples studied by SQUID magnetometry.

The antiferromagnetic character of the IEC in EuS-SrS-SrS trilayers is also clearly observed as a nonmonotonic temperature dependence of the total magnetic moment of the trilayer $M(T)$ measured at weak applied magnetic field. Figure 4 shows the characteristic example of the temperature dependence of magnetization for $\operatorname{EuS}(3.5 \mathrm{~nm})-\mathrm{SrS}(0.7 \mathrm{~nm})-\operatorname{EuS}(3.5 \mathrm{~nm})$ trilayer. The decrease of the total magnetization of the trilayer with the decreasing temperature observed in the case of applied 


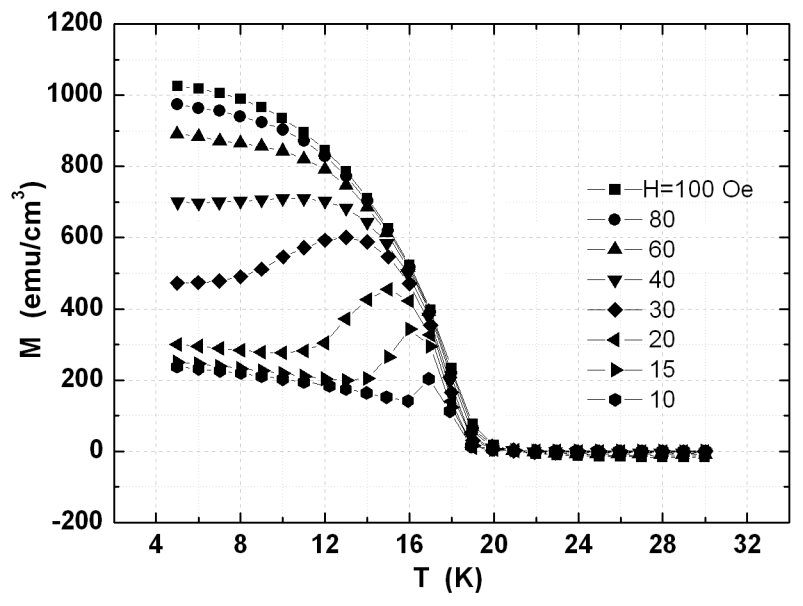

Fig. 4. Temperature dependence of the total magnetic moment of the $\operatorname{EuS}(3.5 \mathrm{~nm})-\operatorname{SrS}(0.7 \mathrm{~nm})-\operatorname{EuS}(3.5 \mathrm{~nm})$ trilayer for various external magnetic fields applied along the [110] easy magnetization axis in the (001) plane of the multilayer.

magnetic fields below about 50 Oe can be qualitatively understood taking into account the temperature dependence of the IEC energy $E_{\text {ex }}(T)=-J(T) M^{2}(T)$. As the exchange coupling mechanism is related to spin splitting of the electronic band structure [16] the exchange integral is also expected to be proportional to the layer magnetization $J \sim M(T)$ [7]. For applied fields stronger than 100 Oe, the temperature behavior of the magnetization follows the usual mean-field-type dependence because the magnetostatic energy $E_{Z}=-H M$ is bigger than the IEC energy over the entire temperatures range studied.

In conclusion, we studied experimentally the magnetic properties of semiconductor trilayers composed of ferromagnetic layers of EuS and ultrathin nonmagnetic insulating SrS space layer. The SQUID magnetometry study of the field and temperature dependences of magnetization revealed the antiferromagnetic alignment of magnetization vectors of EuS layers in EuS-SrS-EuS trilayers with very thin $\mathrm{SrS}$ spacers $(0.5-1 \mathrm{~nm})$. The switching from antiferromagnetic to ferromagnetic alignment was achieved in the external field of the order of $20-50$ Oe. The strength of the antiferromagnetic interlayer coupling (up to $J=-20 \mu \mathrm{J} / \mathrm{m}^{2}$ ) was determined based on model magnetization calculations. These experimental findings provide a basis for future studies of tunneling magnetoresistance and spin-optoelectronic semiconductor heterostructures.

\section{Acknowledgments}

This work was supported in Poland by the European Commission Initial Training Network SemiSpinNet (PITN-GA-2008-215368).

\section{References}

[1] P. Wachter, in: Handbook on the Physics and Chemistry of Rare Earth, Vol. 2, Eds. K.A. Gschneidner, L.R. Eyring, North Holland, Amsterdam 1979, p. 507.

[2] H. Kępa, C.F. Majkrzak, A.Yu. Sipatov, A.G. Fedorov, T.A. Samburskaya, T.M. Giebultowicz, J. Phys., Condens. Matter 21, 124207 (2009).

[3] A. Stachow-Wójcik, T. Story, W. Dobrowolski, M. Arciszewska, R.R. Gałązka, H.J.M. Swagten, W.J.M. de Jonge, A. Twardowski, A.Yu. Sipatov, Phys. Rev. B 60, 15220 (1999).

[4] T. Story, Phys. Status Solidi B 236, 310 (2003).

[5] M. Szot, L. Kowalczyk, P. Deptuła, V. Domukhovski, V. Osinniy, E. Smajek, A. Szczerbakow, A.Yu. Sipatov, V.V. Volobuev, A.G. Fedorov, T. Story, Acta Phys. Pol. A 112, 419 (2007).

[6] H. Kepa, J. Kutner-Pielaszek, J. Blinowski, A. Twardowski, C.F. Majkrzak, T. Story, P. Kacman, R.R. Gałazka, K. Ha, H.J.M. Swagten, W.J.M. de Jonge, A.Yu. Sipatov, V.V. Volobuev, T.M. Giebultowicz, Europhys. Lett. 56, 54 (2001).

[7] C.J.P. Smits, A.T. Filip, H.J.M. Swagten, B. Koopmans, W.J.M. de Jonge, M. Chernyshova, L. Kowalczyk, K. Grasza, A. Szczerbakow, T. Story, W. Palosz, A.Yu. Sipatov, Phys. Rev. B 69, 224410 (2004).

[8] P. Sankowski, H. Kępa, P. Kacman, A.Yu. Sipatov, C.F. Majkrzak, T.M. Giebultowicz, Acta Phys. Pol. A 105, 607 (2004).

[9] L. Kowalczyk, S. Wrotek, P. Dziawa, V. Osinniy, M. Szot, T. Story, A.Yu. Sipatov, V.V. Volobuev, A.G. Fedorov, Acta Phys. Pol. A 110, 225 (2006).

[10] H. Kępa, J. Kutner-Pielaszek, A. Twardowski, C.F. Majkrzak, J. Sadowski, T. Story, T.M. Giebultowicz, Phys. Rev. B 64, 121302(R) (2001).

[11] W. Szuszkiewicz, E. Dynowska, B. Hennin, F. Ott, M. Jouanne, J.F. Morhange, Acta Phys. Pol. A 100, 335 (2001).

[12] J.K. Furdyna, J. Leiner, X. Liu, M. Dobrowolska, S. Lee, J.-H. Chung, B.J. Kirby, Acta Phys. Pol. A 121, 973 (2012).

[13] P. Sankowski, P. Kacman, Phys. Rev. B 71, 201303(R) (2005).

[14] K. Szałowski, T. Balcerzak, Phys. Rev. B 79, 214430 (2009).

[15] V.K. Dugaev, V.I. Litvinov, W. Dobrowolski, T. Story, Solid State Commun. 110, 351 (1999).

[16] J. Blinowski, P. Kacman, Phys. Rev. B 64, 045302 (2001). 